\title{
Diaphragmastimulation hat einen dramatischen negativen Effekt
}

Fragestellung: Ist die elektrische Diaphragmastimulation, die bereits von der US-amerikanischen Food and Drug Administration (FDA) vorläufig zugelassen wurde, um die respiratorische Insuffizienz bei der amyotrophen Lateralsklerose (ALS) therapeutisch zu beeinflussen, wirklich wirksam?

Hintergrund: Seit Jahrzehnten wird überlegt, ob die elektrische Stimulation von motorischen Nerven oder Muskeln bei Vorderhornerkrankungen einen therapeutischen Effekt haben könnte. Es war ein guter Anlass, dieses Prinzip systematisch zu untersuchen; die FDA hatte das NeuRx RA/4 Stimulationssystem des Diaphragmas aus humanitären Gründen zumindest vorübergehend zugelassen. Die Elektroden des NeuRx RA/4 Systems werden mittels eines Zwei-

DiPALS Writing Committee, on behalf of the DiPALS Study Group. Collaborators Safety and efficacy of diaphragm pacing in patients with respiratory insufficiency due to amyotrophic lateral sclerosis (DiPALS): a multicentre, open-label, randomised controlled trial. Lancet Neurol 2015; 14: 883-92 höhleneingriffs direkt auf dem Zwerchfell des Patienten angebracht.

Patienten und Methodik: Es wurde eine Multicenter, Open-Label, randomisierte, kontrollierte klinische Studie durchgeführt. Die Einschlusskriterien waren Stan- dardkriterien für die ALS-Diagnose, der primäre Endpunkt war das Gesamtüberleben. In die Studie wurden 74 Patienten eingeschlossen. Eine Gruppe $(n=37)$ wurde standardmäßig mit nicht invasiver Beatmung behandelt, die andere Gruppe erhielt eine Kombination aus nichtinvasiver Beatmung und dem Zwerchfellstimulator.

Ergebnisse: Die Ergebnisse waren sehr eindeutig: Die Patienten, bei denen zusätzlich zur nichtinvasiven Beatmung das Diaphragma stimuliert wurde, überlebten im Median elf Monate, während die Patienten mit nichtinvasiver Beatmung allein doppelt solange, nämlich 22,5 Monate, überlebten. Insgesamt verstarben 28 von 37 Patienten der Gruppe mit Diaphragmastimulation und nur 19 von 37 der Kontrollgruppe im Beobachtungszeitraum.

Auch die Bilanz der Nebenwirkungen sprach sehr deutlich gegen die Diaphragmastimulation: Während in dieser Gruppe 162 unerwünschte Ereignisse registriert wurden, waren es nur 81 in der Gruppe, die ausschließlich nichtinvasiv beatmet wurde.

Schlussfolgerungen: Eine Diaphragmastimulation hat einen ausgeprägt negativen Effekt auf den Verlauf der ALS; sie sollte bei dieser Erkrankung nicht angewendet werden.

\section{- Kommentar von Albert C. Ludolph, Ulm}

\section{Man sollte besser verschiedene Physiotherapie-„Dosierungen“ prüfen}

Das Ergebnis dieser Studie kann nur eine Lehre auf lange Zeit darstellen: Es ist zunächst erstaunlich, dass die FDA einen Zwerchfellstimulator vorläufig zugelassen hatte, dessen Anwendung eine risikoreiche Zweihöhlenoperation voraussetzte, ohne dass bis dahin auch nur ansatzweise irgend ein publizierter Wirksamkeitsnachweis vorlag. Es beruhigt etwas, dass man nur 74 Patienten brauchte, um diesen Ansatz klar und überzeugend zu widerlegen. Eine noch nicht publizierte französische Studie zeigt ähnliches; es ist zu fordern, dass eine derzeit laufende nordamerikanische Studie unverzüglich abgebrochen wird. Es bleiben allerdings Fragen: Warum konnte man diese bisher negativste (nach der Applikation von intrathekalem BDNF) Interventionsstudie bei der ALS nicht früher beenden? Welche experimentellen Hinweise gab es für den Nutzen einer kontinuierlichen Stimulation des neuromuskulären Apparates bei der ALS? Oder andersherum:

Wir haben als Kliniker den ALS-Patienten immer geraten, den kranken neuromuskulären Apparat zwar moderat zu beanspruchen, aber keinesfalls zu trainieren, da wir aus experimentellen Untersuchungen zu wissen glauben, dass die Vorderhornzelle im Laufe der Erkrankung energetisch erschöpft ist. Ich glaube, dass diese Studie zeigt, dass dieser Ratschlag besser ist, als risikoreich, kostenträchtig, dramatisch und unkritisch ein „device" zu implantieren. Ich halte es für wichtiger, in einer klinischen Studie verschiedene "Dosierungen" von Physiotherapie zu untersuchen. Zu guter Letzt bin ich erleichtert, dass die deutschen ALS-Zentren und das ALS-Netzwerk über Jahre gegenüber den Angeboten und Verlockungen der Hersteller des Zwerchfellstimulators kritisch und standhaft geblieben sind.

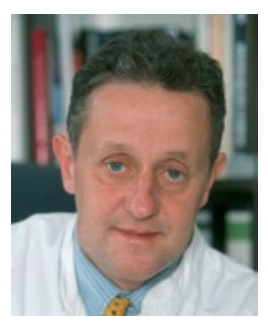

Prof. Dr. med. Albert C. Ludolph, Ulm

Ärztlicher Direktor, Klinik für Neurologie,

Universitätsklinikum UIm

E-Mail: albert.ludolph@rku.de 